\title{
Optimization with Partial Differential Equations in Dieudonné-Rashevsky Form and Conjugate Problems
}

\author{
LAMBERTO Cesari
}

\section{1.}

In previous papers [ $\mathrm{l} a \mathrm{~b} \mathrm{~cd}$ e] we have given existence theorems for problems of optimization with partial differential equations and the usual constraints. In particular, in [1d e] we have given existence theorems for the problem of the minimum of an integral

$$
\begin{gathered}
I[z, u]=\int_{G} F(t, z, u) d t, \\
t=\left(t^{1}, \ldots, t^{\nu}\right), \quad d t=d t^{1} \ldots d t^{v}, \quad z=\left(z^{1}, \ldots, z^{n}\right), \quad u=\left(u^{1}, \ldots, u^{m}\right),
\end{gathered}
$$

in a fixed domain $G \subset E_{\mathrm{v}}$ with differential equations (side conditions) written in the Dieudonné-Rashevsky form

$$
\frac{\partial z^{i}}{\partial t^{j}}=f_{i j}(t, z, u), \quad t \in G, i=1, \ldots, n, j=1, \ldots, v,
$$

usual boundary conditions, and constraints $u \in U(t, z) \subset E_{m}$, where the control space $U=U(t, z)$ is a given subset of $E_{m}$. Here $z=\left(z^{1}, \ldots, z^{n}\right)$ are state variables and $u=\left(u^{1}, \ldots, u^{m}\right)$ control variables.

As stated by P. K. RAshEvsKY [6], very general partial differential equations and systems can be written locally in the form (2).

For instance, second order partial differential equations such as $z_{t}^{1} \pm z_{s s}^{1}=$ $f\left(t, s, z^{1}, z_{t}^{1}, z_{s}^{1}, u\right)$ can be immediately written in the form

$$
z_{t}^{1}=z^{2}, \quad z_{s}^{1}=z^{3}, \quad z_{t}^{2}=v, \quad z_{s}^{2}=w, \quad z_{t}^{3}=w, \quad z_{s}^{3}=\mp v \pm f\left(t, s, z^{1}, z^{2}, z^{3}, u\right),
$$

where $z^{1}, z^{2}, z^{3}$ are state variables and $u, v, w$ are control variables, $m=3, n=3$, $v=2$. Other examples are in [5a b c], [1 d e], and some further ones are given at the end of this paper. Systems of the form (2) have been studied by J. DiEUDONNÉ [2a b], E. DuBINSKY [3], and others, from a completely different viewpoint, as particular cases of systems written in the form

$$
\frac{d z}{d t}=f(t, z, u)
$$

where $z, t$ range in suitable Banach spaces, and $d z / d t$ is the Fréchet derivative of $z$ with respect to $t$.

In [4a] A. I. EgORov has considered abstract problems of optimization with "differential equations" (side conditions) written in the abstract form

$$
A z=f(z, u),
$$

23 Arch. Rational Mech. Anal., Vol. 33 
where $z, u, f$ range in Banach spaces, and $A$ is a linear, generally unbounded, operator. A. I. EGOROV has shown that, whenever we can write a suitable linear problem which is "conjugate" to the given problem of optimization with equation (3) as side conditions, then abstract forms of the minimum property follow (Pontryagin's necessary condition).

In the present paper we give suitable forms for the "conjugate linear problem" for problems of optimization with differential equations in the DieudonnéRashevsky form (2). Furthermore, in harmony with A. I. Egorov [4a], we prove the minimum property in local form, under various sets of hypotheses. A. I. EGOROV's general assumptions are replaced here by somewhat weaker ones in the present situation. For instance, the solutions of the given equations as well as of the conjugate problems need not be unique, and certain continuity hypotheses of EGOROV's paper need be verified only under a coarser topology for the range space $\left(L_{p}\right.$ instead of $\left.W_{p}^{1}\right)$.

For the sake of simplicity we shall limit ourselves to the case $v=2$. Thus, we shall write $(x, y)$ for $t$, and $f_{1}, \ldots, f_{n}, g_{1}, \ldots, g_{n}$ for $f_{i j}$. The results below can be extended immediately to the case $v>2$.

2.

Let $G$ be a Sobolev domain in the $x y$-plane $E_{2}$, and let us consider the class $\Omega$ of all pairs $z(x, y)=\left(z^{1}, \ldots, z^{n}\right), u(x, y)=\left(u^{1}, \ldots, u^{m}\right)$, with $z^{i} \in W_{p}^{1}(G), p \geqq 1$ (thus, $\left.z^{i} \in L_{p}(G), \partial z^{i} / \partial x, \partial z^{i} / \partial y \in L_{p}(G)\right),(i=1, \ldots, n), u(x, y) \in U \subset E_{m}, u^{j}$ measurable in $G, j=1, \ldots, m$, where $U$ is a fixed subset of $E_{m}$, satisfying differential equations and boundary conditions as follows. We require that the pairs $z, u$ in $\Omega$ satisfy the $2 n$ first order partial differential equations

$$
\partial z^{i} / \partial x=f_{i}(x, y, z, u), \quad \partial z^{i} / \partial y=g_{i}(x, y, z, u) \text { a.e. in } G
$$

$(i=1, \ldots, n)$, where $f_{i}, g_{i}$ are real valued continuous functions on $G \times E_{m} \times U$. Let $B=\partial G$ denote the boundary of $G$, and let us assume that $B$ can be divided into finitely many nonoverlapping parts $s_{h}, h=1, \ldots, N$, which we shall call arcs $s$ of $B$, or sides $s$ of $G$. We shall assume the usual conventions concerning the orientation of the components of $B$.

Let $\sigma$ denote the arc length parameter on $B$ starting from fixed arbitrary points of $B, 0 \leqq \sigma \leqq L$, where $L$ is the total Jordan length of $B$. Then $\sigma=\sigma(x, y)$ for $(x, y) \in B$ (with obvious conventions), and we denote by $z^{i}(\sigma)$, or $z^{i}(\sigma(x, y))$, the values of $z^{i}$ for $(x, y) \in B, i=1, \ldots, n$.

We require that the pairs $z, u$ in $\Omega$ satisfy the boundary conditions

$\left\{\begin{array}{l}\text { For each } h=1, \ldots, N \text { and for every } i \text { of a collection }\{i\}_{h} \text { of indices } i=1, \ldots, n \\ \text { depending on } h \text { (which may be empty), let }\end{array}\right.$ \{

$$
z^{i}(\sigma)=\phi_{h i}(\sigma) \quad \text { for } \sigma \in s_{h} \quad \text { (a.e.). }
$$

Here the $\phi_{h l}$ are functions on $s_{h}$ of class $L_{p}\left(s_{h}\right)$. (See Section 6 for alternate boundary conditions.) 
We shall consider the problem of the minimum in $\Omega$ of the functional

$$
I[z, u]=\sum_{h=1}^{N} \sum_{i=1}^{n} \int_{s_{h}}\left[P_{h i}(\sigma) z^{i}(\sigma) d x+Q_{h i}(\sigma) z^{i}(\sigma) d y\right],
$$

where $P_{h i}(\sigma), Q_{h i}(\sigma)$ are given functions $P_{h i}, Q_{h i} \in L_{q}\left(s_{h}\right)$, for some $q \geqq 1$, $q^{-1}+p^{-1} \leqq 1 ; h=1, \ldots, N ; i=1, \ldots, n$.

Let us denote by $t=t(\sigma)$ the tangent vector along the oriented arc $s_{h}$ and by $\cos \widehat{t x}, \cos \widehat{t y}$ the cosines directors of $t$; hence $d x=(\cos \widehat{t x}) d \sigma, d y=(\cos \widehat{t y}) d \sigma$. Let

so that

$$
\Pi_{h i}(t)=P_{h i}(\sigma) \cos (\widehat{t x})+Q_{h i}(\sigma) \cos (\widehat{t y}), \quad i=1, \ldots, n,
$$

$$
I[z, u]=\sum_{h=1}^{N} \sum_{i=1}^{n} \int_{s_{h}} \Pi_{h i}(\sigma) z^{i}(\sigma) d \sigma .
$$

If a side $s_{h}$ of $G$ is a segment parallel to the $y$-axis $\left[x\right.$-axis], then $P_{h i}\left[Q_{h i}\right]$ can be given arbitrary values. We may assume them to be identically zero. Boundary conditions (5) and the expression (6) for the functional do not interfere, that is, we assume that, for every pair $h, i$ with $i \in\{i\}_{h}$, the corresponding functions $P_{h i}, Q_{h i}$ are identically zero.

The case in which the functional is given in the Lagrange form (1) can be reduced to the situation above by suitable transformations which are given in Section 10 for an interval.

As usual we say that a pair $z, u$ in $\Omega$ is optimal for the functional (6) if $I[z, u] \leqq I[\tilde{z}, \tilde{u}]$ for every pair $\tilde{z}, \tilde{u}$ in $\Omega$.

\section{The Hamiltonian}

If $\lambda=\left(\lambda_{1}, \ldots, \lambda_{n}\right), \mu=\left(\mu_{1}, \ldots, \mu_{n}\right)$ denote any two real vector variables, and $(x, y) \in G, z=\left(z^{1}, \ldots, z^{n}\right) \in E_{n}, u=\left(u^{1}, \ldots, u^{m}\right) \in U$, we define the Hamiltonian $H$ as the real-valued function defined in $G \times U \times E_{3 n}$.

$$
H(x, y, z, u, \lambda, \mu)=\sum_{j=1}^{n}\left[\lambda_{j} f_{j}(x, y, z, u)+\mu_{j} g_{j}(x, y, z, u)\right] .
$$

We shall consider different sets of hypotheses below. In each we shall be able to use the first order partial derivatives $\partial f_{i} / \partial z^{j}=f_{i z^{j}}, \partial g_{i} / \partial z^{j}=g_{i z^{j}}, i, j=1, \ldots, n$, and hence also the first order partial derivatives $\partial H / \partial z^{i}, i=1, \ldots, n$, of $H$.

\section{The Linear Conjugate Problem}

We shall denote by $\lambda(x, y)=\left(\lambda_{1}, \ldots, \lambda_{n}\right), \mu(x, y)=\left(\mu_{1}, \ldots, \mu_{n}\right)$ any pair of functions defined in $G, \lambda_{i}, \mu_{i} \in W_{q}^{1}(G), i=1, \ldots, n$. We shall denote by $\Lambda_{h i}(\sigma)$ the expression

$$
\Lambda_{k i}(\sigma)=-\mu_{i}(\sigma) \cos (\widehat{t x})+\lambda_{i}(\sigma) \cos (\widehat{t y}), \quad \sigma \in s_{h},
$$


where $i=1, \ldots, n ; s=1, \ldots, N$. We shall assume that $\lambda_{i}, \mu_{i}, i=1, \ldots, n$, satisfy the system of $n$ first order partial differential equations

$$
\frac{\partial \lambda_{i}}{\partial x}+\frac{\partial \mu_{i}}{\partial y}=-\frac{\partial H}{\partial z^{i}}, \quad i=1, \ldots, n,(x, y) \in G \text { (a.e.) }
$$

with boundary conditions

$$
\Lambda_{h i}(\sigma)=\Pi_{h i}(\sigma), \quad \sigma \in s_{h} \text { (a.e.) }
$$

for every $h=1, \ldots, n$, and for every $i$ not in $\{i\}_{h}$. Note that we do not assign $\Lambda_{h i}$ on sides $s_{h}$ for $i \in\{i\}_{h}$. Note that if a side $s$ is a segment parallel to the $x$-axis then $\cos (\widehat{t y})=0, \cos (\widehat{t x})= \pm 1$, and (9) reduces to

$$
-\mu_{i}(\sigma)=P_{h i}(\sigma), \quad \sigma \in s_{h}, i \notin\{i\}_{h} .
$$

If a side $s$ is a segment parallel to the $y$-axis, then $\cos (\widehat{t x})=0, \cos (\widehat{t y})= \pm 1$, and (9) reduces to

$$
\lambda_{i}(\sigma)=Q_{h i}(\sigma), \quad \sigma \in s_{h}, i \notin\{i\}_{h} .
$$

We say that (8), (9) is the conjugate problem. System (8), or $\partial \lambda_{i} / \partial x+\partial \mu_{i} / \partial y=-\sum_{j}\left[\lambda_{j} f_{j z^{i}}(x, y, z(x, y), u(x, y))+\mu_{j} g_{j z^{i}}(x, y, z(x, y), u(x, y))\right]$, $(i=1, \ldots, n)$ is linear in $\lambda, \mu$. Here $z(x, y), u(x, y)$ denotes any given pair in $\Omega$. Actually, we need consider (8), (9) only when, $z, u$ is a given optimal pair.

We shall assume below that, for a given optimal pair $z, u$, the conjugate problem (8), (9) has at least one solution $\lambda, \mu$ (with $\lambda_{i}, \mu_{i} \in W_{q}^{1}(G), i=1, \ldots, n$ ).

A pair $z, u$ in $\Omega$, together with a solution $\lambda, \mu$ of the corresponding conjugate problem, is said to satisfy the minimum property (Pontryagin's necessary condition in local form) provided that

$$
H(x, y, z(x, y), u(x, y), \lambda(x, y), \mu(x, y)) \leqq H(x, y, z(x, y), u, \lambda(x, y), \mu(x, y))
$$

for almost all $(x, y) \in G$ and all $u \in U$.

\section{Formula for Increments}

If $z, u$ is a pair in $\Omega$, if $\lambda, \mu$ is a solution of the corresponding conjugate problem, and if $z_{\varepsilon}, u_{\varepsilon}$ denotes any pair in $\Omega$, then

where

$$
\begin{aligned}
\Delta I & =I\left[z_{\varepsilon}, u_{\varepsilon}\right]-I[z, u] \\
& =\sum_{h=1}^{N} \sum_{i=1}^{n} \int_{s_{h}} \Pi_{h i}(\sigma)\left(z_{\varepsilon}^{i}(\sigma)-z^{i}(\sigma)\right) d \sigma \\
& =\sum_{h=1}^{N}\left(\sum_{i \in\{i\}_{h}}+\sum_{i \notin\{i\}_{h}}\right) \int_{s_{h}} \Pi_{h i}\left(z_{\varepsilon}^{i}-z^{i}\right) d \sigma,
\end{aligned}
$$

$$
\begin{array}{ll}
\Pi_{h i}=0, & z_{\varepsilon}^{i}=z^{i}, \quad A_{i}\left(z_{\varepsilon}^{i}-z^{i}\right)=0 \\
\Pi_{h i}=\Lambda_{h i} & \text { for } \sigma \in s_{h}, i \notin\{i\}_{h} .
\end{array}
$$


Then

$$
\begin{aligned}
\Delta I & =\sum_{h=1}^{N}\left(\sum_{i \in(i\}_{h}}+\sum_{i \notin\{i\}_{h}}\right) \int_{S_{h}} \Lambda_{h i}\left(z_{\varepsilon}^{i}-z^{i}\right) d \sigma \\
& =\sum_{h=1}^{N} \sum_{i=1}^{n} \int_{s_{h}}\left[-\mu_{i}(\sigma)\left(z^{i}(\sigma)-z^{i}(\sigma)\right) d x+\lambda_{i}(\sigma)\left(z_{\varepsilon}^{i}(\sigma)-z^{i}(\sigma)\right) d y\right],
\end{aligned}
$$

and by Green's theorem

$$
\begin{aligned}
\Delta I= & \sum_{i=1}^{n} \iint_{G}\left[\lambda_{i}\left(\frac{\partial z_{\varepsilon}^{i}}{\partial x}-\frac{\partial z^{i}}{\partial x}\right)+\mu_{i}\left(\frac{\partial z_{\varepsilon}^{i}}{\partial y}-\frac{\partial z^{i}}{\partial y}\right)\right] d x d y \\
& +\sum_{i=1}^{n} \iint_{G}\left(\frac{\partial \lambda_{i}}{\partial x}+\frac{\partial \mu_{i}}{\partial y}\right)\left(z_{\varepsilon}^{i}-z^{i}\right) d x d y=\Lambda_{1}+\Lambda_{2} .
\end{aligned}
$$

If we denote by $f_{i \varepsilon}, g_{i \mathrm{~s}}$ the functions $f_{i}, z_{i}$ where the pair $z(x, y), u(x, y)$ is replaced by $z_{\varepsilon}(x, y), u_{\varepsilon}(x, y)$, then $\Lambda_{1}$ becomes

$$
\begin{aligned}
& \Lambda_{1}= \iint_{G} \sum_{i}\left[\lambda_{i}\left(f_{i \varepsilon}-f_{i}\right)+\mu_{i}\left(g_{i \varepsilon}-g_{i}\right)\right] d x d y \\
&=\iiint_{G}\left[H\left(x, y, z_{\varepsilon}, u_{\varepsilon}, \lambda, \mu\right)-H(x, y, z, u, \lambda, \mu)\right] d x d y \\
&=\iint_{G}\left\{H\left(x, y, z, u_{\varepsilon}, \lambda, \mu\right)-H(x, y, z, u, \lambda, \mu)\right] \\
& \quad+\sum_{i}\left(\partial H(x, y, z, u, \lambda, \mu) / \partial z^{i}\right)\left(z_{\varepsilon}^{i}-z^{i}\right) \\
&\left.\quad+\sum_{i}\left[\frac{\partial H}{\partial z^{i}}\left(x, y, \tilde{z}, u_{\varepsilon}, \lambda, \mu\right)-\frac{\partial H}{\partial z^{i}}(x, y, z, u, \lambda, \mu)\right]\left(z_{\varepsilon}^{i}-z^{i}\right)\right\} d x d y, \\
&=\Lambda_{11}+\Lambda_{12}+\Lambda_{13},
\end{aligned}
$$

where $\tilde{z}=\tilde{z}(x, y)$ denotes a point between $z(x, y)$ and $z_{\varepsilon}(x, y)$ in $E_{n}$.

By formulas (12), (13), and comparison with (8), we obtain $\Lambda_{2}+\Lambda_{12}=0$ and

$$
\Delta I=\Lambda_{11}+\Lambda_{13}=\iint_{G}\left[H\left(x, y, z, u_{\varepsilon}, \lambda, \mu\right)-H(x, y, z, u, \lambda, \mu)\right] d x d y+\eta,
$$

where

$$
\eta=\Lambda_{13}=\iint_{G} \sum_{i}\left[\frac{\partial H}{\partial z^{i}}\left(x, y, \tilde{z}, u_{\varepsilon}, \lambda, \mu\right)-\frac{\partial H}{\partial z^{i}}(x, y, z, u, \lambda, \mu)\right]\left[z_{\varepsilon}^{i}-z^{i}\right] d x d y .
$$

\section{Alternate Boundary Conditions}

We shall now take into consideration boundary conditions for the original problem which are more general than (5). Namely, we shall consider conditions of the form

$$
\sum_{i \in\{i\}_{h}} \alpha_{h i}(\sigma) z^{i}(\sigma)=\phi_{h}(\sigma), \quad \sigma \in s_{h} \text { (a.e.) }
$$


for given functions $\alpha_{h i}$ in $L_{p^{\prime}}\left(s_{h}\right), p^{\prime} \geqq p$, and given functions $\phi_{h}$ in $L_{k}\left(s_{h}\right)$ with $1 / k=1 / p+1 / p^{\prime}$. Precisely, we shall assume that for every $h=1, \ldots, N$ a corresponding collection $\{i\}_{h}$ is assigned as before (which may be empty), and that for every $h$, one or more relations (16) are required, say $v$ relations

$$
\sum_{i \in\{i\}_{h}} \alpha_{h i}^{(\gamma)}(\sigma) z^{i}(\sigma)=\phi_{h \gamma}(\sigma), \quad \sigma \in s_{h} \text { (a.e.) }, \gamma=1, \ldots, v .
$$

As before, we assume that the boundary conditions (17) and the expression (6) for the functional do not interfere, that is, we assume that for every pair, $h, i$ with $i \in\{i\}_{h}$, the corresponding functions $P_{h i}, Q_{h i}$ are identically zero.

The boundary conditions (17) contain conditions (5) as a particular case. Indeed, if $\mu$ denotes the number of elements in the collection $\{i\}_{h}$, we may well require on $s_{h}$ that the $\mu$ relations are satisfied

$$
z^{i}(\sigma)=\phi_{h i}(\sigma), \quad \sigma \in s_{h}, i \in\{i\}_{h},
$$

all of the form (16), namely $\alpha_{h j}=0$ for $j \neq i, \alpha_{h i}=1, \alpha_{h i} \in L_{\infty}, k=p, \phi_{h}=\phi_{h i} \in L_{p}$. These are exactly conditions (5).

Under the new conventions we shall define the conjugate problem by means of the same linear partial differential equations (8) in the unknown functions $\lambda_{i}(x, y), \mu_{i}(x, y), i=1, \ldots, n$, as in Section 4 , with the following extended set of boundary conditions

$$
\Lambda_{h i}(\sigma)=\Pi_{h i}(\sigma), \quad \sigma \in s_{h}(\text { a.e. }), i \notin\{i\}_{h},
$$

and

$$
\left\{\begin{array}{c}
\Lambda_{h i}(\sigma), \sigma \in s_{h}, i \in\{i\}_{h}, \text { are so chosen that for every } \sigma \in s_{h} \text { and any two systems } \\
\text { of numbers } z^{i}(\sigma), i \in\{i\}_{h}, \bar{z}^{i}(\sigma), i \in\{i\}_{h}, \text { satisfying relations }(17) \text { we have also } \\
\qquad \sum_{i \in(i\}_{h}} \Lambda_{h i}(\sigma)\left(z^{i}(\sigma)-\bar{z}^{i}(\sigma)\right)=0 .
\end{array}\right.
$$

Conditions (18a) are the same as conditions (9). On the other hand, under conditions (5), $z^{i}(\sigma)=\bar{z}^{i}(\sigma)=\phi_{h i}(\sigma)$ for $\sigma \in s_{h}$ and $i \in\{i\}_{h}$; hence conditions (18b) are identically satisfied. Thus, we see that whenever the new boundary conditions (17) reduce to conditions (5), then the conjugate problem reduces to the one considered in Section 4. Note that wherever on $B=\partial G$ conditions (17) determine the boundary values of the functions $z^{i}$ then conditions $(18 \mathrm{~b})$ are identically satisfied and do not represent any further restriction on the boundary values of $\lambda_{i}, \mu_{i}$.

In the present more general situation, with conditions (17), (18 a b) replacing (5), (9), we can repeat the argument of Section 5. Indeed, instead of (11), we have now

$$
\begin{gathered}
\Pi_{h i}=0 \quad \text { for } s \in s_{h}, i \in\{i\}_{h}, \\
\sum_{i \in\left\{()_{h}\right.} \Lambda_{h i}\left(z_{\varepsilon}^{i}-z^{i}\right)=0 \text { for } s \in s_{h} ; \quad \Pi_{h i}=\Lambda_{h i} \text { for } \sigma \in s_{h}, i \in\{i\}_{h} .
\end{gathered}
$$

The remaining argument of Section 5 holds now with no further changes. Let us consider examples of conditions (17) other than (5), and corresponding conditions (18b). 
For instance, for $\mu=2$ and (17) reduced to $z^{1}(\sigma)-z^{2}(\sigma)=\phi_{h}(\sigma), \sigma \in s_{h}$ (hence $\left.\alpha_{h 1}=1, \alpha_{h 2}=-1\right)$, then requirement (18b) actually reduces to $\Lambda_{h_{2}}(\sigma)=-\Lambda_{h_{1}}(\sigma)$. Indeed,

$$
\begin{aligned}
\Lambda_{h 1}\left(z^{1}-\bar{z}^{1}\right)+\Lambda_{h 2}\left(z^{2}-\bar{z}^{2}\right) & =\Lambda_{h 1}\left(z^{1}-\bar{z}^{1}\right)-\Lambda_{h 1}\left(z^{2}-\bar{z}^{2}\right) \\
& =\Lambda_{h 1} \phi-\Lambda_{h 1} \phi=0 .
\end{aligned}
$$

For instance, for $\mu=3$ and (17) reduced to the two conditions

$$
z^{1}(\sigma)+z^{2}(\sigma)+z^{3}(\sigma)=0, \quad 2 z^{1}(\sigma)+3 z^{2}(\sigma)+z^{3}(\sigma)=0, \quad \sigma \in S_{h},
$$

then requirement (18b) reduces to $\Lambda_{h 1}, \Lambda_{h 2}, \Lambda_{3}$ orthogonal to $-2,1,1$.

In general, for every $\mu \geqq 1$, if we require, say, on $z^{1}, \ldots, z^{\mu}$ a certain number $v, 1 \leqq v<\mu$, of linear homogeneous relations of the form

$$
\sum_{i=1}^{\mu} \alpha_{h i}^{(\gamma)}(\sigma) z^{i}(\sigma)=0, \quad \gamma=1, \ldots, v,
$$

then relations $(18 \mathrm{~b})$ reduce to

$$
\sum_{i=1}^{\mu} \Lambda_{h i}(\sigma) z^{i}(\sigma)=0
$$

for all systems of numbers $z^{1}(\sigma), \ldots, z^{\mu}(\sigma)$ satisfying the $v$ linear homogeneous relations above. As in Sections 2 and 4 we shall assume that the pairs $z, u$ of the nonempty class $\Omega$ satisfy the boundary value problem (4), (17), and that for a given pair $z, u$ in $\Omega$ there are functions $\lambda_{i}, \mu_{i}$ as stated satisfying the boundary value problem (8), (18a b).

\section{The Case of an Interval Domain}

For an interval domain, say $G=[0 \leqq x \leqq a, 0 \leqq y \leqq b]$, we may prefer to write the functional (6) in the form

$$
\begin{aligned}
I[z, u]= & \sum_{i=1}^{n}\left[\int_{0}^{a}\left(P_{i}(x) z^{i}(x, b)+Q_{i}(x) z^{i}(x, 0)\right) d x\right. \\
& \left.+\int_{0}^{b}\left(R_{i}(y) z^{i}(a, y)+S_{i}(y) z^{i}(0, y)\right) d y\right],
\end{aligned}
$$

where $P_{i}, Q_{i}, R_{i}, S_{i}$ are given functions, $P_{i}, Q_{i} \in L_{q}(0, a), R_{i}, S_{i} \in L_{q}(0, b), q \geqq 1$, $p^{-1}+q^{-1} \leqq 1$.

Let us assume $N=4$ and $s_{1}=[0 \leqq x \leqq a, y=b], s_{2}=[0 \leqq x \leqq a, y=0], s_{3}=$ $[x=a, 0 \leqq y \leqq b], s_{4}=[x=0,0 \leqq y \leqq b]$, the four sides of $G$. Now $s_{1}, s_{2}, s_{3}, s_{4}$ are oriented in the sense of increasing $x$ or $y$. For every $h=1,2,3,4$, and side $s_{h}$ of $G$ let $\{i\}_{h}$ denote any collection of indices $i=1, \ldots, n$. The boundary conditions (5) for the original problem are now of the form

$$
z^{i}=\phi_{h i} \quad \text { on } \quad s_{h}, \quad i \in\{i\}_{h}, h=1,2,3,4 .
$$


The corresponding boundary conditions (9) for the conjugate problem can now be written in the form

$$
\begin{array}{ll}
\mu_{i}(x, b)=P_{i}(x), i \notin\{i\}_{1} ; & \mu_{i}(x, 0)=-Q_{i}(x), i \notin\{i\}_{2} ; \\
\lambda_{i}(a, y)=R_{i}(y), i \notin\{i\}_{3} ; & \lambda_{i}(0, y)=-S_{i}(y), i \notin\{i\}_{4} .
\end{array}
$$

As expected, we need not assign the values of any $\lambda_{i}\left[\mu_{i}\right]$ on sides $y=0$, or $y=b[x=0$, or $x=a]$, and of course we do not assign the values of $\lambda_{i}, \mu_{i}$ on sides $s_{h}$ if $i \in\{i\}_{h}, i=1, \ldots, n, h=1,2,3,4$.

Note that in the present situation and with the boundary conditions (20) and (21), identity (11) of Section 5 can be proved by integration by parts, while identity (12), written in the present notations, can be directly verified.

Let us now assume that boundary conditions (19) are replaced by more general boundary conditions (17) for the original problem. Conditions (17) will now be written in the form

$$
\sum_{i \in\{i\}_{h}} \alpha_{h i}^{\gamma} z^{i}=\phi_{h \gamma} \quad \text { on } \quad s_{h}, \quad \gamma=1, \ldots, v, h=1,2,3,4,
$$

where $v$ as usual may well depend on $h$, and $\alpha_{h i}^{\gamma}, \phi_{h \gamma}$ are known functions on $s_{h}$ as in Section 6. Let us write the corresponding boundary conditions for the conjugate problem. Conditions (18a) are now replaced by conditions (20). Conditions (18b) become

$$
\begin{array}{ll}
\sum_{i \in\{i\}_{1}} \mu_{i}(x, b)\left(z^{i}(x, b)-\bar{z}^{i}(x, b)\right)=0, & 0 \leqq x \leqq a, \\
\sum_{i \in\{i\}_{2}} \mu_{i}(x, 0)\left(z^{i}(x, 0)-\bar{z}^{i}(x, 0)\right)=0, & 0 \leqq x \leqq a, \\
\sum_{i \in\{i\}_{3}} \lambda_{i}(a, y)\left(z^{i}(a, y)-\bar{z}^{i}(a, y)\right)=0, & 0 \leqq y \leqq b, \\
\sum_{i \in\{i\}_{4}} \lambda_{i}(0, y)\left(z^{i}(0, y)-\bar{z}^{i}(0, y)\right)=0, & 0 \leqq y \leqq b,
\end{array}
$$

where, in each equation, $z^{i}, \bar{z}^{i}, i \in\{i\}_{h}$, denote any two solutions of the corresponding equation (22), $h=1,2,3,4$.

In the present situation $-G$ an interval of the $x y$-plane, $s_{h}, h=1,2,3,4$, the four sides of $G$ - we can take into consideration boundary conditions even slightly more general than (22) for the original problem. Indeed, we may require instead of (22), that

$$
\begin{array}{ll}
\sum_{i \in\{i\}_{1}} \alpha_{h i}^{\gamma}(x) z^{i}(x, b)+\sum_{i \in\{i\}_{2}} \beta_{h i}^{\gamma}(x) z^{i}(x, 0)=\phi_{\gamma}(x), & 0 \leqq x \leqq a, \gamma=1, \ldots, v^{\prime}, \\
\sum_{i \in\{i\}_{3}} \gamma_{h i}^{\gamma}(y) z^{i}(a, y)+\sum_{i \in\{i\}_{4}} \delta_{h i}^{\gamma}(y) z^{i}(0, y)=\psi_{\gamma}(y), & 0 \leqq y \leqq a, \gamma=1, \ldots, v^{\prime \prime},
\end{array}
$$

where $v, v^{\prime \prime}$ are two integers, and $\alpha_{h i}^{\gamma}, \beta_{h i}^{\gamma}, \gamma_{h i}^{\gamma}, \delta_{h i}^{\gamma}, \phi_{h}, \psi_{y}$ are given functions on $[0, a]$ or $[0, b]$ analogous to the functions $\alpha_{h i}^{\gamma}, \phi_{h y}$ of equations (17). Let us write now the corresponding boundary conditions for the conjugate problem. Conditions (18a) again are replaced by conditions (21). Conditions (18b) are now 
replaced by relations

$$
\begin{aligned}
& \sum_{i \in\{i\}_{1}} \mu_{i}(x, b)\left(z^{i}(x, b)-\bar{z}^{i}(x, b)\right)+\sum_{i \in\{i\}_{2}} \mu_{i}(x, 0)\left(z^{i}(x, 0)-\bar{z}^{i}(x, 0)\right)=0, \\
& \sum_{i \in\{i\}_{3}} \lambda_{i}(a, y)\left(z^{i}(a, y)-\bar{z}^{i}(a, y)\right)+\sum_{i \in\{i\}_{4}} \lambda_{i}(0, y)\left(z^{i}(0, y)-\bar{z}^{i}(0, y)\right)=0,
\end{aligned}
$$

where $z^{i}(x, b), i \in\{i\}_{1}, z^{i}(x, 0), i \in\{i\}_{2}$, and $\bar{z}^{i}(x, b), i \in\{i\}_{1}, \bar{z}^{i}(x, 0), i \in\{i\}_{2}$ denote any two systems of numbers satisfying the first equations (24), and an analogous convention hold for the numbers $z^{i}(a, y), i \in\{i\}_{3}, z^{i}(0, y), i \in\{i\}_{4}$, and $\bar{z}^{i}(a, y)$, $i \in\{i\}_{3}, \bar{z}^{i}(0, y), i \in\{i\}_{4}$.

For instance, if relations (24) are

$$
z^{i}(x, b)=z^{i}(x, 0), \quad z^{i}(a, y)=z^{i}(0, y), \quad i=1, \ldots, n,
$$

then relations (25) can be written in the form

$$
\mu_{i}(x, b)=\mu_{i}(x, 0), \quad \lambda_{i}(a, y)=\lambda_{i}(0, y), \quad i=1, \ldots, n .
$$

Under the new boundary conditions (24), (21), (25), the proofs in Section 5 are essentially the same as above.

\section{Hypotheses}

For the sake of simplicity, in this and following Sections we shall be concerned with the problems (original and conjugate) as worded in Sections 2 and 4 . We leave it to the reader to make the obvious changes for the alternate problems of Sections 6 and 7.

We shall consider different sets of independent hypotheses, say $\left(H_{1}\right)$, or $\left(\mathrm{H}_{2}\right)$, or $\left(\mathrm{H}_{3}\right)$ below. In any case we make the following general assumptions:

( $\alpha$ ) For a given pair $z, u$ in $\Omega$ (a given optimal pair in the proof of the necessary condition), the linear conjugate problem (9), (10) has some solution $\lambda, \mu$, (with $z^{i} \in W_{p}^{1}(G), \quad i=1, \ldots, n, u^{j}$ measurable, $j=1, \ldots, m, \lambda_{i}, \mu_{i} \in W_{q}^{1}(G), \quad i=1, \ldots, n$, $\left.q^{-1}+p^{-1} \leqq 1\right)$.

( $\beta$ ) There are pairs $z_{\varepsilon}, u_{\varepsilon}$ in $\Omega$ for every $u_{\varepsilon}$ obtained by modifying $u$ as follows: take any point $(\bar{x}, \bar{y})$ in the interior of $G$, a circle $R$ of center $(\bar{x}, \bar{y})$ and radius $r>0$ with $R \subset G$, any point $\overline{\bar{u}} \in U$, any measurable subset $E$ of $R$, and take $u_{\varepsilon}(x, y)=$ $u(x, y)$ in $G-E, u_{\varepsilon}(x, y)=\overline{\bar{u}}$ in $E$.

We list now the alternate specific hypotheses:

$\left(H_{1}\right)$ (linear case). Here we assume $p \geqq 1$ and

(26) $f_{i}=\sum_{j} A_{i j}(x, y) z^{j}+C_{i}(x, y, u), g_{j}=\sum_{j} B_{i j}(x, y) z^{i}+D_{i}(x, y, u), \quad i=1, \ldots, n$,

where the functions $A_{i j}, B_{i j}$ are continuous in $G$, the functions $C_{i}, D_{i}$ are continuous in $G \times U$, and $U$ is compact.

Actually, much less is needed. Indeed, $U$ may be any subset of $E_{m}$, and we need require only that the functions $A_{i j}, B_{i j}$ are in $L_{q}(G), q^{-1}+p^{-1} \leqq 1$; that the functions $C_{i}, D_{i}$ are measurable in $x, y$ for every $u \in U$, with $C_{i}, D_{i} \in L_{q}(G)$ for 
every fixed $u \in U$;

$$
\iint_{G}\left|C_{i}\right|^{q} d x d y, \quad \iint_{G}\left|D_{i}\right|^{q} d x d y \leqq M
$$

for some constant $M$ which may depend on $u$, and such that for almost all $(\bar{x}, \bar{y}) \in G$ and every fixed $u \in U$ we have

$$
\left(\pi r^{2}\right)^{-1} \iint_{R} C_{i}(x, y, u) d x d y \rightarrow C_{i}(\bar{x}, \bar{y}, u), \quad i=1, \ldots, n,
$$

as $r \rightarrow 0+$; and that the same holds for $D_{i}, i=1, \ldots, n$. For $p=1$ we understand that (27) is replaced by boundedness of $C_{i}, D_{i}$ in $G, i=1, \ldots, n$, for every fixed $u \in U$.

$\left(\mathrm{H}_{2}\right)$ (nonlinear case). We assume here $p=q=2$. We assume that the functions $f_{i}, g_{i}$ are continuous in $G \times E_{n} \times U$ with continuous partial derivatives $\partial f_{i} / \partial z^{j}=f_{i z^{j}}$, $\partial g_{i} / \partial z^{j}=g_{i z}{ }^{j}, i, j=1, \ldots, n$, and that these first order partial derivatives are Lipschitzian with respect to $z$ and $u$ of some constant $K$ (and exponent one) in $G \times U \times E_{n}$. In addition to $(\beta)$ we assume that, for every $u_{\varepsilon}$ as mentioned, there is at least one $z_{\varepsilon}$ with $\left(z_{\varepsilon}, u_{\varepsilon}\right) \in \Omega$ and $\left\|z_{\varepsilon}-z\right\|_{L_{p}} \leqq L\left\|u_{\varepsilon}-u\right\|_{L_{1}}$ for some fixed constant $L$. We assume that the given functions $P_{i}, Q_{i}, R_{i}, S_{i}$ are essentially bounded. In addition to $(\alpha)$ we assume that $u, \lambda, \mu$ are essentially bounded in $G$, or $|u(x, y)| \leqq m,|\lambda(x, y)|$, $|\mu(x, y)| \leqq m^{\prime}$ for almost all $(x, y) \in G$ and some $m, m^{\prime} \geqq 0$ (though the derivatives $\lambda_{i x}, \lambda_{i y}, \mu_{i x}, \mu_{i y}, i=1, \ldots, n$, may well be unbounded and belong to $L_{q}(G)$ ).

$\left(\mathrm{H}_{3}\right)$ (nonlinear case). Here we make the same assumptions as in $\left(\mathrm{H}_{2}\right)$, where the first order partial derivatives $f_{i z^{j}}, g_{i z^{j}}, i, j=1, \ldots, n$, are assumed to be Lipschitzian with respect to $z$ and $u$ with some constant $K$ and exponent $\alpha, 0<\alpha<1$. Here we take $p>1, q>1$ with $(1+\alpha) p^{-1}+q^{-1}=1$. No boundedness assumptions for $\lambda, \mu$ are assumed here.

Remark. Other sets of conditions can be taken into consideration, where $u$ can be assumed to be not essentially bounded, and $p$ can be taken equal to one. (See, for instance, [7] for a hyperbolic system of partial differential equations.)

\section{A Necessary Condition for a Minimum}

Theorem. Under hypotheses $\left(H_{1}\right)$, if $z, u$ is a given pair in $\Omega$, if $\lambda, \mu$ is a solution of the corresponding linear conjugate problem, then $z, u$ is optimal if and only if $z, u, \lambda, \mu$ satisfy the minimum property. Under hypotheses $\left(\mathrm{H}_{2}\right)$, or $\left(\mathrm{H}_{3}\right)$, if $z, u$ is a given optimal pair in $\Omega$, and if $\lambda, \mu$ is a solution of the corresponding linear conjugate problem, then $z, u, \lambda, \mu$ satisfy the minimum property.

Proof. (a) Let us assume that hypotheses $\left(H_{1}\right)$ hold, and let us prove the sufficiency of the condition. Indeed under these hypotheses we have $\eta=0$ and (14) yields

$$
\Delta I=\iint_{G}\left[H\left(x, y, z, u_{\varepsilon}, \lambda, \mu\right)-H(x, y, z, u, \lambda, \mu)\right] d x d y
$$

for every pair $z_{\varepsilon}, u_{\varepsilon}$ of $\Omega$ (and $z$ does not appear in (29)). Then (10) certainly implies that the term in brackets in (29) is $\geqq 0$ for almost all $(x, y) \in G$. Hence $\Delta I \geqq 0$, or $I\left[z_{\varepsilon}, u_{\varepsilon}\right] \geqq I[z, u]$. The sufficiency property is proved (under hypotheses $\left.\left(H_{1}\right)\right)$. 
(b) Again, let us assume that hypothesis $\left(H_{1}\right)$ holds, and let us prove the necessity of the condition. For any point $(\bar{x}, \bar{y})$ interior to $G$ we shall denote by $R$ a circle of center $(\bar{x}, \bar{y})$ and radius $r$ with $R \subset G$. Then $\varepsilon=\pi r^{2}$ is the area of $R$. If $\overline{\bar{u}}$ denotes any point of $U$, then by $u_{\varepsilon}(x, y)$ we shall denote the function defined by taking $u_{\varepsilon}(x, y)=u(x, y)$ in $G-R$ and by $u_{\varepsilon}(x, y)=\overline{\bar{u}}$ in $R$. Let $z_{\varepsilon}(x, y)$ be a function $z$ so that the pair $z_{\varepsilon}, u_{\varepsilon}$ is in $\Omega$. Then $\eta=0$, and (14) yields

$$
0 \leqq \Delta I=\iint_{G}\left[H\left(x, y, z, u_{\varepsilon}, \lambda, \mu\right)-H(x, y, z, u, \lambda, \mu)\right] d x d y,
$$

where the bracket is zero in $G-R$. Then

$$
0 \leqq \varepsilon^{-1} \Delta I=\varepsilon^{-1} \iint_{R}\left[H\left(x, y, z, u_{\varepsilon}, \lambda, \mu\right)-H(x, y, z, u, \lambda, \mu)\right] d x d y,
$$

and because of (26) also

$$
\begin{aligned}
0 \leqq \varepsilon^{-1} \Delta I= & \varepsilon^{-1} \iint_{R}\left[\sum_{i} \lambda_{i}(x, y)\left(C_{i}(x, y, \overline{\bar{u}})-C_{i}(x, y, u(x, y))\right)\right. \\
& \left.+\sum_{i} \mu_{i}(x, y)\left(D_{i}(x, y, \overline{\bar{u}})-D_{i}(x, y, u(x, y))\right)\right] d x d y .
\end{aligned}
$$

If $U$ is compact and the functions $C_{i}(x, y, u), D_{i}(x, y, u)$ are continuous in the compact set $G \times U$, hence bounded in $G \times U$, the expressions

$$
\sum_{i} \lambda_{i}(x, y) C_{i}(x, y, u(x, y)), \quad \sum_{i} \mu_{i}(x, y) D_{i}(x, y, u(x, y))
$$

are $L_{q^{-}}$, hence $L_{1}$-integrable in $G$. By a theorem of Lebesgue there is a subset $E$ of points $(\bar{x}, \bar{y})$ interior to $G$ with meas $(G-E)=0$, such that

$$
\begin{aligned}
& \varepsilon^{-1} \iint_{R} \sum_{i} \lambda_{i} C_{i} d x d y \rightarrow \sum_{i} \lambda_{i}(\bar{x}, \bar{y}) C_{i}(\bar{x}, \bar{y}, u(\bar{x}, \bar{y})) \\
& \varepsilon^{-1} \iint_{R}\left|\lambda_{i}(x, y)-\lambda_{i}(\bar{x}, \bar{y})\right| d x d y \rightarrow 0, \quad i=1, \ldots, n,
\end{aligned}
$$
as $\varepsilon \rightarrow 0+$ for every $(\bar{x}, \bar{y}) \in E$, and the same holds for the functions $\sum_{i} \mu_{i} D_{i}$ and
$\mu_{i}, i=1, \ldots, n$, respectively. On the other hand

$$
\begin{aligned}
\varepsilon^{-1} \iint_{R} \sum_{i} \lambda_{i}(x, y) C_{i}(x, y, \overline{\bar{u}}) d x d y-\sum_{i} \lambda_{i}(\bar{x}, \bar{y}) C_{i}(\bar{x}, \bar{y}, \overline{\bar{u}}) \\
=\varepsilon^{-1} \iint_{R}\left|\lambda_{i}(x, y)-\lambda_{i}(\bar{x}, \bar{y})\right| C_{i}(x, y, \overline{\bar{u}}) d x d y \\
\quad+\lambda_{i}(\bar{x}, \bar{y}) \cdot \varepsilon^{-1} \iint_{R}\left|C_{i}(x, y, \overline{\bar{u}})-C_{i}(\bar{x}, \bar{y}, \bar{u})\right| d x d y=\Delta_{1}+\Delta_{2} .
\end{aligned}
$$

Because of the boundedness and continuity of the functions $C_{i}$, we conclude that $\Lambda_{1} \rightarrow 0, \Delta_{2} \rightarrow 0$ as $\varepsilon \rightarrow 0$ for every $(\bar{x}, \bar{y}) \in E$ and $\overline{\bar{u}} \in U$, and the same holds for $\mu_{i}, D_{i}$ replacing $\lambda_{i}, C_{i}$. From (30) we conclude that

$$
\begin{aligned}
\varepsilon^{-1} \Delta I \rightarrow & \sum_{i} \lambda_{i}(\bar{x}, \bar{y})\left(C_{i}(\bar{x}, \bar{y}, \overline{\bar{u}})-C_{i}(\bar{x}, \bar{y}, u(\bar{x}, \bar{y}))\right) \\
& +\sum_{i} \mu_{i}(\bar{x}, \bar{y})\left(D_{i}(\bar{x}, \bar{y}, \overline{\bar{u}})-D_{i}(\bar{x}, \bar{y}, u(\bar{x}, \bar{y}))\right) \geqq 0
\end{aligned}
$$


for all $(\bar{x}, \bar{y}) \in E$ and $\overline{\bar{u}} \in U$, or

$$
\begin{aligned}
H(\bar{x}, \bar{y}, z & (\bar{x}, \bar{y}), \overline{\bar{u}}, \lambda(\bar{x}, \bar{y}), \mu(\bar{x}, \bar{y})) \\
& -H(\bar{x}, \bar{y}, z(\bar{x}, \bar{y}), u(\bar{x}, \bar{y}), \lambda(\bar{x}, \bar{y}), \mu(\bar{x}, \bar{y})) \geqq 0
\end{aligned}
$$

for the same $(\bar{x}, \bar{y}) \in E$ and $\overline{\bar{u}} \in U$.

If $U$ is not necessarily compact and $C_{i}, D_{i}$ are not necessarily continuous, but (27), (28) hold, first we note that functions (31) are certainly $L_{1}$-integrable in $G$ as differences between $f_{i}(x, y, z(x, y), u(x, y))=\partial z^{i} / \partial x$ and $\sum_{j} A_{i j}(x, y) z^{j}(x, y)$, and between $g_{i}=\partial z^{i} / \partial y$ and $\sum_{j} B_{i j} z^{j}$, respectively. By the same Lebesgue theorem we can choose $E$ in such a way that (32) still holds together with the analogous relation for $\mu_{i}, D_{i}$, in such a way that (28) holds at every $(\bar{x}, \bar{y}) \in E$, and in such a way that

$$
\varepsilon^{-1}\left(\iint_{R}\left|\lambda_{i}(x, y)-\lambda_{i}(\bar{x}, \bar{y})\right|^{q} d x d y \rightarrow 0, \quad i=1, \ldots, n,\right.
$$

as $\varepsilon \rightarrow 0$ for every $(\bar{x}, \bar{y}) \in E$, together with the analogous relations for the functions $\mu_{i}, i=1, \ldots, n$. Then, using (27), (28) we conclude that again $\Delta_{1} \rightarrow 0, \Delta_{2} \rightarrow 0$ for every $(x, y) \in E$ and all $\overline{\bar{u}} \in U$. As before we conclude that (33) holds for every $(\bar{x}, \bar{y}) \in E$ and all $\overline{\bar{u}} \in U$. We have proved the necessity of the condition under hypotheses $\left(H_{1}\right)$.

(c) Let us prove the necessity of the condition under hypotheses $\left(H_{2}\right)$. Suppose that the statement is not true. Then, there is a set $E \subset G$ of positive measure where (10) is not true (for all $\overline{\bar{u}} \in U$ ). The functions $z, u, \lambda, \mu$ are measurable (and $L_{1^{-}}$ integrable) in $G$; hence the set $F$ of the points $(x, y) \in G$ of asymptotic continuity has full measure. Hence $E \cap F$ has also positive measure, and we take a point $(\bar{x}, \bar{y}) \in E \cap F$ interior to $G$. Since $(\bar{x}, \bar{y}) \in E$, there is some $\overline{\bar{u}} \in U$ and number $h>0$ such that

$$
H(\bar{x}, \bar{y}, \bar{z}, \overline{\bar{u}}, \bar{\lambda}, \bar{\mu})=H(\bar{x}, \bar{y}, \bar{z}, \bar{u}, \bar{\lambda}, \bar{\mu})-h
$$

where $\bar{z}=\bar{z}(\bar{x}, \bar{y}), \bar{u}=u(\bar{x}, \bar{y}), \bar{\lambda}=\lambda(\bar{x}, \bar{y}), \bar{\mu}=\mu(\bar{x}, \bar{y})$. Since $(\bar{x}, \bar{y}) \in F$ and is interior to $G$, there is a set $E_{0} \subset G$ of density one at $(\bar{x}, \bar{y})$ such that

$H(x, y, z(x, y), \overline{\bar{u}}, \lambda(x, y), \mu(x, y)) \leqq H(x, y, z(x, y), u(x, y), \lambda(x, y), \mu(x, y))-h / 2$

for all $(x, y) \in E_{0}$. We can choose $r>0$ so that the circle $R$ of center $(\bar{x}, \bar{y})$ and radius $r$ is interior to $G$, and such that meas $\left(E_{0} \cap R\right)$ : meas $R>1 / 2$. If $\varepsilon=$ meas $R=\pi r^{2}$, then we have meas $\left(E_{0} \cap R\right)>\varepsilon / 2$.

Let us define the function $u_{\varepsilon}(x, y),(x, y) \in G$, by taking $u_{\varepsilon}(x, y)=u(x, y)$ in $G-E_{0} \cap R$, and $u_{\varepsilon}(x, y)=\overline{\bar{u}}$ in $E_{0} \cap R$. Then

$$
\begin{aligned}
H\left(x, y, z, u_{\varepsilon}, \lambda, \mu\right)-H(x, y, z, u, \lambda, \mu) & =0 & & \text { in } G-E_{0} \cap R, \\
& \leqq-h / 2 & & \text { in } E_{0} \cap R,
\end{aligned}
$$

and the integral in the expression (14) of $\Delta I$ is then $\leqq(-h / 2)$ meas $\left(E_{0} \cap R\right) \leqq$ $-h \varepsilon / 4$. On the other hand, we can take $m, m^{\prime}$ so that $|u|,\left|u_{\varepsilon}\right| \leqq m,|\lambda|,|\mu| \leqq m^{\prime}$ in $G$, and then

$$
\iint_{G}\left|u_{\varepsilon}(x, y)-u(x, y)\right| d x d y \leqq 2 m \text { meas } R=2 m \varepsilon .
$$


If $z_{\varepsilon}(x, y),(x, y) \in G$, denotes a solution of (4), (5) satisfying the requirement stated in $\left(\mathrm{H}_{2}\right)$, then

$$
\left(\iint_{G}\left|z_{\varepsilon}(x, y)-z(x, y)\right|^{2} d x d y\right)^{\frac{1}{2}} \leqq 2 m L \varepsilon .
$$

Finally,

$$
\begin{aligned}
|\eta|= & \mid \iint_{G} \sum_{i j}\left[\lambda_{j}\left(f_{j z^{i}}\left(x, y, \tilde{z}, u_{\varepsilon}\right)-f_{j z^{i}}(x, y, z, u)\right)\right. \\
& \left.+\mu_{j}\left(g_{j z^{i}}\left(x, y, \tilde{z}, u_{\varepsilon}\right)-g_{j z^{i}}(x, y, z, u)\right)\right]\left[z_{\varepsilon}^{i}-z^{i}\right] d x d y \mid \\
\leqq & 2 n^{2} m^{\prime} K \iint_{G}\left(\left|z_{\varepsilon}-z\right|+\left|u_{\varepsilon}-u\right|\right)\left|z_{\varepsilon}-z\right| d x d y \\
\leqq & 2 n^{2} m^{\prime} K\left[\iint_{G}\left|z_{\varepsilon}-z\right|^{2} d x d y+\left(\iint_{G}\left|u_{\varepsilon}-u\right|^{2} d x d y\right)^{\frac{1}{2}}\left(\iint_{G}\left|z_{\varepsilon}-z\right|^{2} d x d y\right)^{\frac{1}{2}}\right] \\
\leqq & 2 n^{2} m^{\prime} K\left[\left(L \iint_{G}\left|u_{\varepsilon}-u\right| d x d y\right)^{2}\right. \\
& \left.+\left(2 m \iint_{G}\left|u_{\varepsilon}-u\right| d x d y\right)^{\frac{1}{2}}\left(L \iint_{G}\left|u_{\varepsilon}-u\right| d x d y\right)\right] \\
\leqq & 2 n^{2} m^{\prime} K\left[L^{2}(2 m \varepsilon)^{2}+(2 m)^{\frac{1}{2}} L(2 m \varepsilon)^{\frac{1}{2}}(2 m \varepsilon)\right] \\
= & 8 n^{2} m^{2} m^{\prime} K L\left(L \varepsilon^{2}+\varepsilon^{\frac{3}{2}}\right) .
\end{aligned}
$$

Relation (14) now yields

$$
0 \leqq \Delta I \leqq-4^{-1} h \varepsilon+8 n^{2} m^{2} m^{\prime} K L\left(L \varepsilon^{2}+\varepsilon^{\frac{3}{2}}\right) .
$$

By taking $r$ sufficiently small (hence $\varepsilon$ sufficiently small), this relation is contradictory. We have proved that (10) holds for almost all $(x, y) \in G$ and all $u \in U$. The theorem is proved under hypotheses $\left(\mathrm{H}_{2}\right)$.

(d) Let us prove the theorem under hypotheses $\left(H_{3}\right)$. The reasoning is the same as in (c) but now (34) is replaced by

$$
\begin{aligned}
|\eta| \leqq & 2 n^{2} K\left[\left(\iint_{G}|\lambda|^{q} d x d y\right)^{1 / q}+\left(\iint_{G}|\mu|^{q} d x d y\right)^{1 / q}\right]\left[\int \int _ { G } \left(\left|z_{\varepsilon}-z\right|^{(\alpha+1) p^{\prime}}\right.\right. \\
& \left.\left.+\left|u_{\varepsilon}-u\right|^{\alpha p^{\prime}}\left|z_{\varepsilon}-z\right|^{p^{\prime}}\right) d x d y\right]^{1 / p^{\prime}},
\end{aligned}
$$

where $p^{\prime}>1$ is defined by $\left(p^{\prime}\right)^{-1}+(q)^{-1}=1$. Then $\left(p^{\prime}\right)^{-1}=(1+\alpha)(p)^{-1}$, or $(1+\alpha) p^{\prime}=p, 1<p^{\prime}<p$. If we take $m^{\prime \prime}=\|\lambda\|_{L_{q}}+\|\mu\|_{L_{q}}$, we have

$$
\begin{aligned}
|\eta| \leqq & 2 n^{2} m^{\prime \prime} K\left[\iint_{G}\left|z_{\varepsilon}-z\right|^{p} d x d y\right. \\
& \left.+\left(\iint_{G}\left|u_{\varepsilon}-u\right|^{p} d x d y\right)^{\alpha /(1+\alpha)}\left(\iint_{G}\left|z_{\varepsilon}-z\right|^{p} d x d y\right)^{p^{\prime} / p}\right]^{1 / p^{\prime}} \\
\leqq & 2 n^{2} m^{\prime \prime} K\left[\left(L \iint_{G}\left|u_{\varepsilon}-u\right| d x d y\right)^{p}\right. \\
& \left.+\left((2 m)^{p-1} \iint_{G}\left|u_{\varepsilon}-u\right| d x d y\right)^{\alpha /(1+\alpha)}\left(L \iint_{G}\left|u_{\varepsilon}-u\right| d x d y\right)^{p^{\prime}}\right]^{1 / p^{\prime}} \\
\leqq & 2 n^{2} m^{\prime \prime} K\left[(2 m L \varepsilon)^{p}+\left((2 m)^{p-1}(2 m \varepsilon)\right)^{\alpha /(1+\alpha)}(2 m L \varepsilon)^{p^{\prime}}\right]^{1 / p^{\prime}} \\
\leqq & K_{1}\left(\varepsilon^{p / p^{\prime}}+\varepsilon^{1+\alpha /(1+\alpha) p^{\prime}}\right)=K_{1}\left(\varepsilon^{1+\alpha}+\varepsilon^{1+\alpha / p}\right)
\end{aligned}
$$


for a suitable constant $K_{1}>0$. Then (35) is replaced by

$$
0 \leqq \Delta I \leqq-4^{-1} h \varepsilon+K_{1}\left(\varepsilon^{1+\alpha}+\varepsilon^{1+\alpha / p}\right),
$$

and the reasoning is now the same as for $\left(\mathrm{H}_{2}\right)$.

\section{The Functional in Lagrange Form}

If the functional is written in the Lagrange form (1), and $G$ is the interval $[0 \leqq x \leqq a, 0 \leqq y \leqq b]$, then

$$
I[z, u]=\int_{0}^{a} \int_{0}^{b} F(x, y, z, u) d x d y,
$$

and the following transformations lead to a functional of the form (19). First, we introduce an auxiliary variable $z^{n+1}$ satisfying the differential equation

$$
\frac{\partial^{2} z^{n+1}}{\partial x \partial y}=F(x, y, z, u)
$$

and boundary conditions $z^{n+1}(x, 0)=0, z^{n+1}(0, y)=0$, and then $I[z, u]=z^{n+1}(a, b)$. Now, let us introduce the variables $z^{n+2}, z^{n+3}$ by taking

$$
\begin{aligned}
& \frac{\partial z^{n+1}}{\partial x}=z^{n+2}, \quad \frac{\partial z^{n+2}}{\partial x}=v, \quad \frac{\partial z^{n+3}}{\partial x}=F(x, y, z, u), \\
& \frac{\partial z^{n+1}}{\partial y}=z^{n+3}, \quad \frac{\partial z^{n+2}}{\partial y}=F(x, y, z, u), \quad \frac{\partial z^{n+3}}{\partial y}=w,
\end{aligned}
$$

where $v, w$ are new control variables. For $z^{n+1}, z^{n+2}, z^{n+3}$ we have the boundary conditions

$$
z^{n+1}(x, 0)=z^{n+2}(x, 0)=0, \quad z^{n+1}(0, y)=z^{n+3}(0, y)=0,
$$

and the functional takes the forms

$$
\begin{aligned}
I=z^{n+1}(a, b) & =\int_{0}^{a} z^{n+2}(x, b) d x=\int_{0}^{b} z^{n+3}(a, y) d y \\
& =2^{-1} \int_{0}^{a} z^{n+2}(x, b) d x+2^{-1} \int_{0}^{b} z^{n+3}(a, y) d y .
\end{aligned}
$$

If we now consider the vector $\tilde{z}=\left(z^{1}, \ldots, z^{n}, z^{n+1}, z^{n+2}, z^{n+3}\right)$ of $n+3$ state variables, and the vector $\tilde{u}=\left(u^{1}, \ldots, u^{m}, v, w\right)$ of $m+2$ control variables, we have a unique Dieudonné-Rashevsky system of $2 n+6$ equations (4) and (37), we have a functional (38) of the form (19), and a control space $U=U \times E_{2}$. Here all $P_{i}, Q_{i}$ are zero but one which is equal to one (or two equal to $\frac{1}{2}$ each). The set $\tilde{U}$ is not bounded even if $U$ is bounded, because, in general, we have no bounds for $v=\partial z^{n+2} / \partial x$ and $w=\partial z^{n+3} / \partial y$. Nevertheless, if we disregard these two equations in the system of $2 n+6$ equations (second and sixth equations (37)), and we take the corresponding multipliers identically to zero (see example (a) below), then $v$ and $w$ will never appear in our discussion, and the results above apply with trivial changes. 


\section{Examples}

(a) The problem of the minimum of $I[z, u]=z(a, b)$ ( $z$ scalar) with differential equation

$$
z_{x y}=f\left(x, y, z, z_{x}, z_{y}, u\right), \quad(x, y) \in G=[0, a ; 0, b],
$$

boundary conditions $z(x, 0)=0, z(0, y)=0$, and constraints $u \in U$ can be written as the problem of the minimum of the functional $I=z^{1}(a, b)$ with differential equations

$$
\begin{array}{lll}
z_{x}^{1}=z^{2}, & z_{x}^{2}=v, & z_{x}^{3}=f\left(x, y, z^{1}, z^{2}, z^{3}, u\right), \\
z_{y}^{1}=z^{3}, & z_{y}^{2}=f\left(x, y, z^{1}, z^{2}, z^{3}, u\right), & z_{y}^{3}=w,
\end{array}
$$

boundary conditions $z^{1}(x, 0)=z^{2}(x, 0)=0, z^{1}(0, y)=z^{3}(0, y)=0$, and constraints $(u, v, w) \in \tilde{U}=U \times E_{2}$. Then the functional

$$
I=z^{1}(a, b)=\int_{0}^{a} z^{2}(x, b) d x=\int_{0}^{b} z^{3}(a, y) d y=2^{-1} \int_{0}^{a} z^{2}(x, b) d x+2^{-1} \int_{0}^{b} z^{3}(a, y) d y
$$

has the form (19) with $P_{1}=0, P_{2}=\frac{1}{2}, P_{3}=0, Q_{1}=Q_{2}=Q_{3}=0, R_{1}=0, R_{2}=0$, $R_{3}=\frac{1}{2}, S_{1}=S_{2}=S_{3}=0$. The Hamiltonian is

$$
H=\lambda_{1} z^{2}+\lambda_{2} v+\lambda_{3} f+\mu_{1} z^{3}+\mu_{2} f+\mu_{3} w
$$

with six multipliers. Nevertheless, it is convenient to disregard the second and sixth equations (33), and correspondingly take $\lambda_{2}=0, \mu_{3}=0$ in $G$. Thus, we have the boundary value problem in $G=[0 \leqq x \leqq a, 0 \leqq y \leqq b]$

$$
\begin{aligned}
& z_{x}^{1}=z^{2}, \quad z_{y}^{1}=z^{3}, \quad z_{y}^{2}=f, \quad z_{x}^{3}=f, \\
& z^{1}(x, 0)=z^{2}(x, 0)=0, \quad z^{1}(0, y)=z^{3}(0, y)=0,
\end{aligned}
$$

and the Hamiltonian has now the reduced form

$$
H=\lambda_{1} z^{2}+\mu_{1} z^{3}+\left(\lambda_{3}+\mu_{2}\right) f,
$$

with four multipliers $\lambda_{1}, \mu_{1}, \lambda_{3}, \mu_{2}$. The control variable is again $u \in U$. The conjugate equations ( 8 ) and the corresponding boundary conditions (21) are now

$$
\begin{aligned}
\frac{\partial \lambda_{1}}{\partial x}+\frac{\partial \mu_{1}}{\partial y} & =-\left(\lambda_{3}+\mu_{2}\right) f_{z}, \\
\frac{\partial \mu_{2}}{\partial y_{1}} & =-\lambda_{1}-\left(\lambda_{3}+\mu_{2}\right) f_{z_{x}}, \\
\frac{\partial \lambda_{3}}{\partial x} \quad & =-\mu_{1}-\left(\lambda_{3}+\mu_{2}\right) f_{z_{y}}, \\
\lambda_{1}(a, y)=0, \quad \mu_{1}(x, b) & =0, \quad \mu_{2}(x, b)=1, \quad \lambda_{3}(a, y)=1 .
\end{aligned}
$$


M. B. Suryanarayana [7] has studied in detail the present problem (with $z$ a vector) and has given sufficient conditions for the existence in Sobolev spaces of solutions to the original boundary value problem (41) and the conjugate boundary value problem (42). Also, he has shown that if $L=\lambda_{3}+\mu_{2}$ is sufficiently smooth and $H^{*}$ denotes the expression $H^{*}=L f$, then $L$ satisfies

$$
\frac{\partial^{2} L}{\partial x \partial y}=H_{z}^{*}-\frac{\partial}{\partial x}\left(H_{z_{x}}^{*}\right)-\frac{\partial}{\partial y}\left(H_{z_{y}}^{*}\right)
$$

with boundary conditions $L_{x}=-\partial H^{*} / \partial z_{y}$ for $y=b, L_{y}=-\partial H^{*} / \partial z_{x}$ for $x=a$, and $L(a, b)=2$. This is the special form of the linear conjugate problem used by A. I. EgoRov in his previous paper [4b] for the problem of the present example.

(b) The problem of the minimum of $I[z, u]==\int_{0}^{a} z(a, y) d y, z$ scalar, with differential equation

$$
z_{x}=z_{y y}+f\left(x, y, z, z_{y}, u\right)
$$

boundary conditions $z(0, y)=0, z(x, 0)=z(x, a)=0$, and constraints $u \in U$, can be written as the problem of the minimum of the functional $I=\int_{0}^{b} z^{1}(a, y) d y$, with differential equations

$$
\begin{aligned}
& z_{x}^{1}=z^{2}, \quad z_{x}^{2}=v, \quad z_{x}^{3}=w, \\
& z_{y}^{1}=z^{3}, \quad z_{y}^{2}=w, \quad z_{y}^{3}=z^{2}-f\left(x, y, z^{1}, z^{3}, u\right),
\end{aligned}
$$

boundary conditions $z^{1}(0, y)=z^{3}(0, y)=0, \quad z^{1}(x, 0)=z^{2}(x, 0)=0, \quad z^{1}(x, b)=$ $z^{2}(x, b)=0$, and constraints $(u, v, w) \in \widetilde{U}=U \times E_{2}$. Then the functional $I$ has the form (19) with $P_{1}=P_{2}=P_{3}=0, Q_{1}=Q_{2}=Q_{3}=0, R_{1}=1, R_{2}=0, R_{3}=0, S_{1}=$ $S_{2}=S_{3}=0$, and the Hamiltonian is

$$
H=\lambda_{1} z^{2}+\lambda_{2} v+\lambda_{3} w+\mu_{1} z^{3}+\mu_{2} w+\mu_{3}\left(z^{2}-f\right) .
$$

It is convenient to disregard in (44) the second, third, and fifth equations, and correspondingly to take $\lambda_{2}=\lambda_{3}=\mu_{2}=0$ in $G$. Thus, we have the boundary value problem in $G$

$$
\begin{aligned}
& z_{x}^{1}=z^{2}, \quad z_{y}^{1}=z^{3}, \quad z_{y}^{3}=z^{2}-f\left(x, y, z^{1}, z^{3}, u\right), \\
& z^{1}(x, 0)=z^{2}(x, 0)=0, \quad z^{1}(x, b)=z^{2}(x, b)=0, \quad z^{1}(0, y)=z^{3}(0, y)=0,
\end{aligned}
$$

and the Hamiltonian has now the reduced form

$$
H=\lambda_{1} z^{2}+\mu_{1} z^{3}+\mu_{3}\left(z^{2}-f\right)
$$


with multipliers $\lambda_{1}, \mu_{1}, \mu_{3}$ and constraints $u \in U$. The conjugate equations (8) and the corresponding boundary conditions (21) are now

$$
\begin{gathered}
\frac{\partial \lambda_{1}}{\partial x}+\frac{\partial \mu_{1}}{\partial y}=\mu_{3} f_{z}, \\
0=-\lambda_{1}-\mu_{3}, \\
\frac{\partial \mu_{3}}{\partial y}=-\mu_{1}+\mu_{3} f_{z_{y}}, \\
\lambda_{1}(a, y)=1, \quad \mu_{3}(x, b)=0, \quad \mu_{3}(x, 0)=0 .
\end{gathered}
$$

Note that if we take $\mu_{3}=-\lambda_{1}$, the Hamiltonian reduces to $H=\lambda_{1} f+\mu_{1} z^{3}$ and the conjugate system to

$$
\frac{\partial \lambda_{1}}{\partial x}+\frac{\partial \mu_{1}}{\partial y}=-\lambda_{1} f_{z}, \quad \frac{\partial \lambda_{1}}{\partial y}=\mu_{1}+\lambda_{1} f_{z_{y}}
$$

with boundary conditions $\lambda_{1}(a, y)=1, \lambda_{1}(x, 0)=\lambda_{1}(x, b)=0$. By eliminating $\mu_{1}$ we obtain (formally) the equation

$$
\frac{\partial \lambda_{1}}{\partial x}=-\frac{\partial^{2} \lambda_{1}}{\partial y^{2}}-\lambda_{1} f_{z}+\frac{\partial}{\partial y}\left(\lambda_{1} f_{z_{y}}\right) .
$$

(c) The problem of the minimum of

$$
I[z, u]=\int_{b}^{a} \int_{0}^{b} f_{0}\left(x, y, z, z_{x}, z_{y}, u\right) d x d y
$$

with differential equation

$$
z_{x x}+z_{y y}=f\left(x, y, z, z_{x}, z_{y}, u\right),
$$

boundary conditions $z(x, 0)=z(x, b)=z(0, y)=z(a, y)=0$, and constraints $u \in U$, can be written as the problem of minimum of the functional $I=z^{1}(a, b)$ with differential equations

$$
\begin{aligned}
& z_{x y}^{1}=f_{0}\left(x, y, z, z_{x}, z_{y}, u\right), \\
& z_{x x}+z_{y y}=f\left(x, y, z, z_{x}, z_{y}, u\right),
\end{aligned}
$$

and boundary conditions $z^{1}(x, 0)=z^{1}(0, y)=0, \quad z(x, 0)=z(x, b)=z(0, y)=$ $z(a, y)=0$. Equivalently, by writing $z^{4}$ for $z$, we shall seek the minimum of the functional

$$
I=z^{1}(a, b)=2^{-1} \int_{0}^{a} z^{2}(x, b) d x+2^{-1} \int_{0}^{b} z^{3}(a, y) d y,
$$

with differential equations

$$
\begin{gathered}
z_{x}^{1}=z^{2}, \quad z_{x}^{2}=v^{\prime}, \quad z_{x}^{3}=f_{0}\left(x, y, z^{4}, z^{5}, z^{6}, u\right), \quad z_{x}^{4}=z^{5}, \quad z_{x}^{5}=v, \quad z_{x}^{6}=w, \\
\text { (48) } z_{y}^{1}=z^{3}, \quad z_{y}^{2}=f_{0}\left(x, y, z^{4}, z^{5}, z^{6}, u\right), \quad z_{y}^{3}=w^{\prime}, \quad z_{y}^{4}=z^{6}, \quad z_{y}^{5}=w, \\
z_{y}^{6}=-v+f\left(x, y, z^{4}, z^{5}, z^{6}, u\right),
\end{gathered}
$$


boundary conditions $z^{1}(x, 0)=z^{2}(x, 0)=0, \quad z^{1}(0, y)=z^{3}(0, y)=0, \quad z^{4}(x, 0)=$ $z^{5}(x, 0)=0, \quad z^{4}(x, b)=z^{5}(x, b)=0, \quad z^{4}(0, y)=z^{6}(0, y)=0, \quad z^{4}(a, y)=z^{6}(a, y)=0$, and constraints $\left(u, v^{\prime}, w^{\prime}, v, w\right) \in \tilde{U}=U \times E_{4}$. We shall denote the twelve multipliers by $\lambda_{1}, \ldots, \lambda_{6}, \mu_{1}, \ldots, \mu_{6}$. It is convenient to disregard the second, sixth, ninth, and eleventh equations of (48), and correspondingly to take $\lambda_{2}=\lambda_{6}=\mu_{3}=$ $\mu_{5}=0$ in $G$. Then the Hamiltonian is

$$
H=\lambda_{1} z^{2}+\lambda_{3} f_{0}+\lambda_{4} z^{5}+\lambda_{5} v+\mu_{1} z^{3}+\mu_{2} f_{0}+\mu_{4} z^{6}+\mu_{6}(-v+f)
$$

with eight multipliers, and the constraints are now $(u, v) \in U \times E_{1}$. Here $P_{1}=0$, $P_{2}=1, P_{3}=0, P_{4}=P_{5}=P_{6}=0, Q_{1}=Q_{2}=Q_{3}=Q_{4}=Q_{5}=Q_{6}=0, R_{1}=0, R_{2}=0$, $R_{3}=1, R_{4}=R_{5}=R_{6}=0, S_{1}=S_{2}=S_{3}=S_{4}=S_{5}=S_{6}=0$. The conjugate problem is now (formally)

where

$$
\begin{aligned}
\lambda_{1 x}+\mu_{1 y} & =0 \\
\mu_{2 y} & =-\lambda_{1} \\
\lambda_{3 x} & =-\mu_{1} \\
\lambda_{4 x}+\mu_{4 y} & =-\left(\lambda_{3}+\mu_{2}\right) f_{0 z}-\mu_{6} f_{z} \\
\lambda_{5 x \quad} \quad & =-\lambda_{4}-\mu_{6} f_{z_{x}}-\left(\lambda_{3}+\mu_{2}\right) f_{0 z_{x}} \\
\mu_{6 y} & =-\mu_{4}-\mu_{6} f_{z_{y}}-\left(\lambda_{3}+\mu_{2}\right) f_{0 z_{y}}
\end{aligned}
$$

$$
\begin{array}{lll}
\lambda_{1}(a, y)=0, & \lambda_{3}(a, y)=1, & \lambda_{5}(a, y)=1 \\
\mu_{1}(x, b)=0, & \mu_{2}(x, b)=1, & \mu_{6}(x, b)=0 \\
\lambda_{5}(0, y)=0, & & \mu_{6}(x, 0)=0 .
\end{array}
$$

We have above six first order partial differential equations in eight unknowns. M. B. Suryanarayana [7] has proved the existence of solutions of the conjugate problem (49).

This research was partially supported by AFOSR grant 942-65.

\section{References}

1. Cesari, L., (a) Existence Theorems for Multidimensional Problems of Optimal Control. Differential Equations and Dynamical Systems. Academic Press 1967, 115-132. (b) Existence theorems for multidimensional Lagrange problems. Journal of Optimization Theory and Applications 1, 87-112 (1967). - (c) Sobolev spaces and multidimensional Lagrange problems of optimization. Annali Scuola Normale Sup. Pisa 22, 193-227 (1968). - (d) Multidimensional Lagrange problems of optimization in a fixed domain and an application to a problem of magnetohydrodynamics. Arch. Rational Mech. Anal. 29, 81 - 104 (1968). - (e) Existence Theorems for Lagrange Problems in Sobolev Spaces. Symposium on Nonlinear Functional Analysis, April 16-18, 1968, Chicago (to appear).

2. Dieudonné, J., (a) Foundations of Modern Analysis. xiv + 361 pp. Academic Press 1960. -

(b) Deux examples singuliers d'équations différentielles. Acta Scientiarum Mathematicarum 12, 38-40 (1950).

3. Dubinsky, E., Differential equations and differential calculus in Montel spaces. Trans. Amer. Math. Soc. 110, 1-21 (1964). 
4. Egorov, A. I., (a) Optimal control in Banach space. Math. Systems Theory 1, 347-352 (1968). - (b) Optimal control processes in certain systems with distributed parameters. Autom. Telem. 25, 5, 613-623 (1964) = Autom. Remote Control 25, 557-567 (1965).

5. LURIE, K. A., (a) The Mayer-Bolza problem for multiple integrals and the optimization of the performance of systems with distributed parameters. Prikl. Mat. Mek 27, 842-853 (1963) = PMM, Pergamon Press, 27, 1284- 1299 (1963). - (b) Optimal control of conductivity of a fluid moving in a channel in a magnetic field. Prikl. Mat. Mek 28, 258-267 (1964) = PMM, Pergamon Press, 28, 316- 327 (1964). - (c) The Mayer-Bolza Problem for Multiple Integrals: Some Optimum Problems for Elliptic Differential Equations Arising in Magnetohydrodynamics. Topics in Optimization. Academic Press 1967, 147 - 193.

6. Rashevsky, P. K., Geometric Theory of Partial Differential Equations [Russian]. GSTZ, Moscow 1947, p. 354.

7. Suryanarayana, M. B., Optimization with Hyperbolic Partial Differential Equations. A doctoral thesis at the University of Michigan, Ann Arbor 1969.

Department of Mathematics University of Michigan Ann Arbor

(Received December 13, 1968) 